\title{
The Capacity of Mycobacterium tuberculosis Complex Species and M. bovis BCG Substrains Specific Identification - Implications for Optimized PCR-Based Diagnostics in Adverse Events Following Vaccination Suspected Cases
}

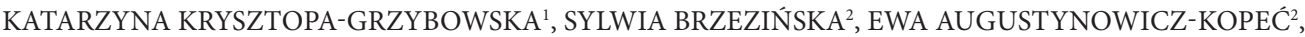 \\ ZOFIA ZWOLSKA ${ }^{2}$, EWA AUGUSTYNOWICZ ${ }^{1}$ and ANNA LUTYŃSKA ${ }^{1 *}$
}

\author{
${ }^{1}$ National Institute of Public Health - National Institute of Hygiene, Warsaw, Poland \\ ${ }^{2}$ National Tuberculosis and Lung Diseases Research Institute, Warsaw, Poland
}

Received 26 April 2012, revised 18 September 2012, accepted 21 September 2012

\section{Abstract}

\begin{abstract}
The capacities of differentiation of Mycobacterium bovis BCG from other members of M. tuberculosis complex species using PCR-RFLP, multiplex PCR, and PCR-based genomic deletion analysis approaches were compared. In the study, mycobacteria isolated from patients suspected of adverse events following vaccination with BCG, primarily classified according presence of RD1 marker as virulent and avirulent mycobacteria, were used. The PCR-based genomic deletion analysis was found the best option for mycobacteria diagnostics improvement, as it was capable precisely differentiate virulent and avirulent mycobacteria or virulent species of $M$. tuberculosis complex. The routine confirmation of mycobacteria species in the cases of adverse events following BCG vaccination is highly expected, especially in clinical practice of patients with primary immunodeficiency.
\end{abstract}

Key words: Mycobacterium bovis BCG, mycobacteria specific identification, PCR-based diagnostics

\section{Introduction}

Although extensive studies have been performed to develop advanced vaccines against tuberculosis (TB), vaccination with attenuated Mycobacterium bovis Bacille Calmette-Guérin (BCG) is the only commercially available vaccine against TB (WHO, 2011). The BCG vaccine is used worldwide (Brewer, 2000) and generally is regarded as being the safest in use, but the real incidence of disease evoked by BCG strains, e.g. BCG-itis or osteitis, is not known (Behr, 2002). Localized abscesses, regional lymphadenopathy, and disseminated disease in immunocompromised hosts are generally regarded as rare but well recognized complications following BCG vaccination (Bernatowska et al., 2007). Abscesses at BCG injection sites and in places other than BCG injection sites have also been described in healthy hosts (Pankowska and Rożniecki, 1997; Gołębiowska et al., 2008). In Poland, BCG vaccination has been a part of the National Immunization Program since 1951, and currently involves a single BCG dose given to neonates only. BCG AEFI (Adverse Events Following Immunization) cases are registered through passive surveillance and most of them are not routinely confirmed in the laboratory. The rate of AEFI in Poland is regarded as low (Szczuka, 2002), however, lack of simple and routine diagnostics does not allow to estimate its real incidence. Lack of routinely used species-specific diagnostics also complicates fast and reliable diagnosis of mycobacteria grown from BCG vaccinated individuals suffering from immunodeficiencies.

The M. tuberculosis complex consists of mycobacteria characterized by high DNA-DNA homology (from hybridization studies) and identical 16S rRNA sequences but differ in phenotypes and pathogenicity. This group has consisted of seven closely related species: M. tuberculosis - infecting human and primates; M. africanum, a heterogeneous group causing human tuberculosis in Africa; M. microti, found as a source of infection in voles and very rarely in immunocompromised humans; M. bovis, causing tuberculosis in cattle and a wide variety of other animals, including man; M. bovis Bacille Calmette-Guérin (BCG), an attenuated vaccine strain; $M$. canetti, a very rare, smooth variant of M. tuberculosis, usually isolated in Africa; and M. caprae, primarily causal agent of caprine TB with transmission to human and wild animals reported (Brosch et al., 2002; Cousins et al., 2003; Rodríguez

\footnotetext{
* Corresponding author: A. Lutyńska, National Institute of Public Health - National Institute of Hygiene, 24 Chocimska Street, 00-791 Warsaw, Poland; phone: +48 225421 213; fax: +48 225421 311; e-mail: alutynska@pzh.gov.pl
} 
et al., 2009). More recently, novel species has been described: M. mungi, pathogen inducing high mortality rates among banded mongooses living in close association with humans in Botswana (Alexander et al., 2010); M. pinnipedii, previously known as seal bacillus primarily infecting pinnipeds but is also pathogenic humans and, possibly, cattle (Cousins et al., 2003) and M. orygis, causative agent of tuberculosis in oryxes, gazelles, and waterbucks in Africa; cows and rhesus monkeys in South Asian; and humans (van Ingen et al., 2012).

It has been estimated that as many as 49 BCG substrains might be used in the world (Corbel et al., 2004) since the original Bacille Calmette-Guérin attenuated strain has been distributed worldwide. BCG daughter strains were found heterogenic as the result of the microevolution due to specific production processes, which can be currently specifically identified with molecular tools (Behr, 2002).

The use of reliable molecular tools applicable for confirmation of species identity in AEFI suspected cases are expected to precisely identify all $M$. tuberculosis complex representatives together with a possibility to identify M. bovis BCG substrains. In order to evaluate the capacities of molecular diagnostic tools described previously as potentially applicable for M. tuberculosis complex identification, in this pilot study we have used reference mycobacteria species and randomly chosen mycobacteria isolates grown from AEFI suspected cases and collected at the National Tuberculosis and Lung Diseases Research Institute (NTLDRI). The mycobacteria isolated from patients and chosen for the purpose of the study have been primarily classified as virulent and avirulent mycobacteria, as they were found habour and not harbour RD1 marker, respectively. Three potentially applicable variants of PCR-based methods involving different species-specific or substrain-specific sequences published previously were introduced for testing and compared.

\section{Experimental}

\section{Material and Methods}

Strains. A total of 10 mycobacterial strains isolated from BCG AEFI suspected cases within 2004-2010, have been included in the study. At NTLDRI they were diagnosed as virulent $(7575,3221,1339,5995,5379)$ and avirulent $(60,4138,868,1078,2714)$ mycobacteria according PCR performed with primers specific for RD1 region (Talbot et al., 1997). All strains were grown on solid Löwenstein-Jensen medium for about 20 days at $37^{\circ} \mathrm{C}$, harvested, heat inactivated. The following reference strains were used: M. bovis BCG Moreau originating from BCG vaccine lot no. 00108, M. bovis BCG
Danish 1331 (NIBSC, 07/270), M. bovis BCG Tokyo 172 (NIBSC, 07/272), M. tuberculosis H37Rv (ATCC 25618), M. bovis (ATCC 19211), M.microti (ATCC 19422), M. africanum (ATCC 25420), M.pinnipedii (ATCC BAA-688), and M. canetti (kindly obtained from Prof. Dr. Dick van Soolingen from Tuberculosis Reference Laboratory, National Institute for Public Health and the Environment, The Netherlands).

DNA extraction. Chromosomal DNA was isolated from mycobacterial strains as described by van Soolingen et al. (1991).

PCR. In the study we have tested PCR-RFLP developed by Kasai et al. (2000), PCR-based genomic deletion analysis described by Warren et al. (2006) and multiplex PCR developed by Bedwell et al. (2001). System developed by Kasai et al. (2000) involved restriction of amplified product of the partial gyrB gene with RsaI or TaqI enzymes. PCR-based genomic deletion analysis involved PCR method with use of primers specific for RD1, RD4, RD9, and RD12 regions (Warren et al., 2006). In a multiplex PCR described by Bedwell et al. (2001) primers specific for RD1, RD2, RD8, RD16 and the senX3-regX3 regions were primarily developed for purposes of identification of different substrains of M. bovis BCG.

\section{Results}

Among 10 mycobacteria isolates, 5 primarily classified at the NTLDRI as avirulent mycobacteria (60, $4138,868,1078,2714$ ), were easily identified as M. bovis BCG Moreau isolates (Fig. 1A) as they showed a multiplex-PCR profiles presence of $196 \mathrm{bp}(\triangle \mathrm{RD} 1), 252 \mathrm{bp}$ (RD14), $276 \mathrm{bp}$ (senX3-regX3), $315 \mathrm{bp}$ (RD2) and the 472 bp (RD8) amplified fragments. Multiplex-PCR profiles of reference M. bovis BCG Danish and M. bovis BCG Tokyo substrains according of size of amplified fragments were easily differentiated from M.bovis BCG Moreau substrain. Other 5 mycobacteria isolates, classified at the NTLDRI as virulent $(7575,3221,1339$, 5995, 5379), presented profiles different from those of $M$. bovis BCG reference strains and samples of avirulent mycobacteria. In the profiles of mycobacteria samples, pre-classified as virulent, there were found products seen in the profiles of all M.tuberculosis complex reference strains. The amplification profiles of virulent mycobacteria samples were composed of fragments RD1 (146 bp), RD14 (252 bp), RD2 (315 bp), RD16 (401 bp), RD8 (472 bp) regions but did not contained the PCR product expected for the senX3-regX3 region. As multiplex PCR profiles did not contained $352 \mathrm{bp}$ and/or $276 \mathrm{bp}$ products, single PCR reactions were performed with senX3-regX3 region primers revealing their presence and suggesting the inhibition 
Fig 1. A, multiplex PCR; B, single reaction for senX3-regX3; C, PCR-based genomic deletion analysis; D, PCR-RFLP gyrB lane 1,60; lane 2, 4138; lane 3, 868; lane 4, 1078; lane 5, 2714; lane 6, 7575; lane 7, 3221; lane 8, 1339; lane 9, 5995; lane 10, 5379; lane 11, M. bovis BCG Moreau; lane 12, M. bovis BCG Danish; lane 13, M. bovis BCG Tokyo; lane 14, M.tuberculosis H37Rv; lane 15, M. bovis; lane 16 , M. africanum; lane 17, M. microti; lane 18 , M. canetti; lane 19, M. pinnipedii; $\mathrm{M}$, molecular marker $100 \mathrm{bp}$.
$\begin{array}{llllllllllllllllllllll}M & 1 & 2 & 3 & 4 & 5 & 6 & 7 & 8 & 9 & 10 & M & 11 & 12 & 13 & 14 & 15 & 16 & 17 & 18 & 19 & M\end{array}$

A

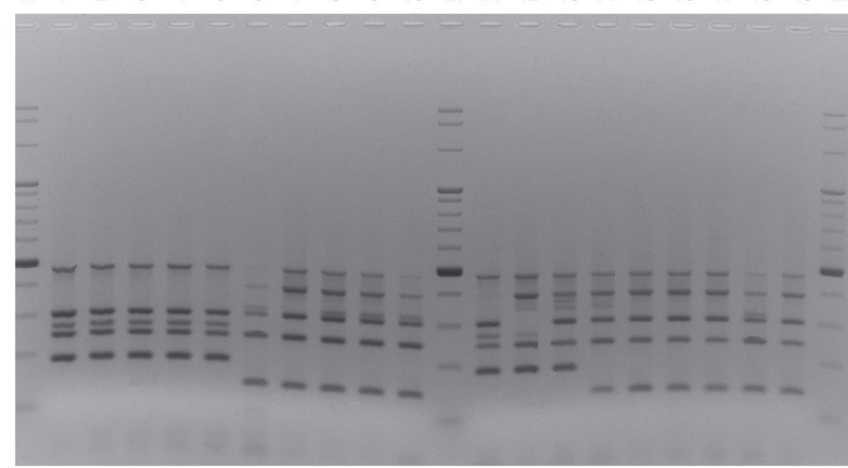

$\begin{array}{llllllllllllllllllllll}M & 1 & 2 & 3 & 4 & 5 & 6 & 7 & 8 & 9 & 10 & M & 11 & 12 & 13 & 14 & 15 & 16 & 17 & 18 & 19 & M\end{array}$

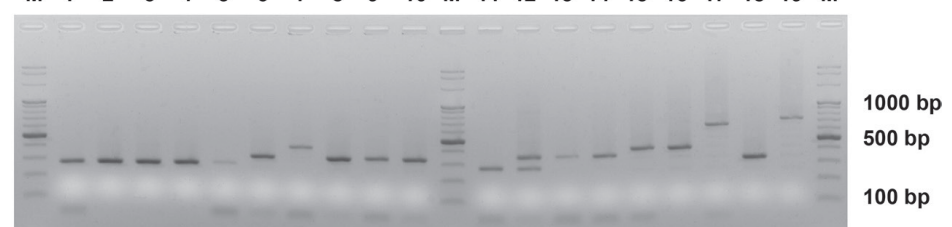

$\begin{array}{llllllllllllllllllllll}M & 1 & 2 & 3 & 4 & 5 & 6 & 7 & 8 & 9 & 10 & M & 11 & 12 & 13 & 14 & 15 & 16 & 17 & 18 & 19 & M\end{array}$
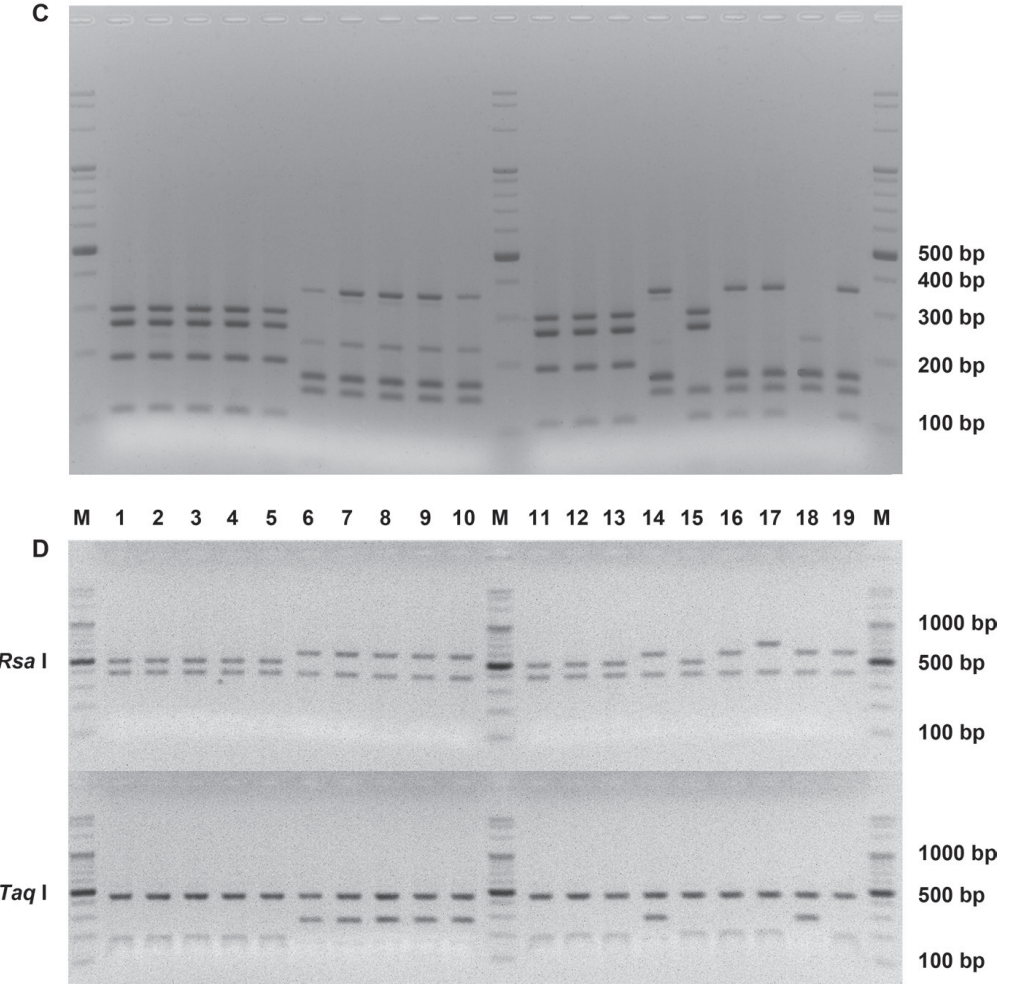

of the PCR reaction in cases of the virulent mycobacteria isolates (Fig. 1B).

During the PCR-based genomic deletion analysis, 5 isolates classified at the NTLDRI as avirulent mycobacteria $(60,4138,868,1078,2714)$, were easily identified as M. bovis BCG (Fig. 1C) as they showed a profile of deletion regions $\mathrm{RD} 9, \mathrm{RD} 1, \mathrm{RD} 4$ and $\mathrm{RD} 12$ (with amplicon sizes: 108 bp, 196 bp, 268 bp and 306 bp, respectively). Theirs profiles were identical with profiles obtained for M. bovis BCG Moreau, M. bovis BCG Danish and M. bovis BCG Tokyo substrains. Other 5 mycobacteria isolates, classified at the NTLDRI as virulent ones (7575, $3221,1339,5995,5379)$, presented profiles specific for M. tuberculosis with presence of amplified fragments 
from RD1, RD4, RD9, RD12 regions (146bp, 172 bp, $235 \mathrm{bp}, 369 \mathrm{bp}$ products, respectively).

In the first step of PCR-RFLP gyrB analysis unique sequence for $M$. tuberculosis complex was amplified. Fragment of $1020 \mathrm{bp}$ was present in all mycobacteria strains used in the study (data not shown) and confirmed affiliation to the M. tuberculosis complex. At the second step, the amplified DNA fragments were digested by RsaI or TaqI and enabled four species of M. tuberculosis, M. africanum, M. bovis and M. microti to be differentiated. As shown in the Fig. 1D, M. bovis and M. microti could be differentiated from the other species by the presence of RsaI-digested fragments of $500 \mathrm{bp}$ and $700 \mathrm{bp}$, respectively. TaqI digestion of the partial gyrB gene generated a $300 \mathrm{bp}$-fragment specific for M. tuberculosis as described Kasai et al. (2000) but also for M. canetti.

\section{Discussion}

The adverse events following vaccination with BCG, although registered by national surveillance after clinical recognition, are confirmed in the laboratory rarely. Moreover, the differentiation of $M$. bovis BCG from other members of the M. tuberculosis complex has previously been regarded difficult (Augustynowicz-Kopeć et al., 2006). Some variants of NAT (Nucleic Acid Amplification Techniques) have been described in order to improve M. tuberculosis complex or M. bovis BCG substrains diagnostics possibilities (Jagielski et al., 2010a, 2010b). In our study we have compared diagnostics capacity of gyrB-based PCR-RFLP, multiplex PCR, and PCR-based genomic deletion analysis using a set of reference mycobacteria strains and ten mycobacteria isolates originating from BCG AEFI suspected cases collected at the NTLDRI.

The first one - the gyrB-based PCR-RFLP method, previously shown to be useful for differentiation of closely related strains of bacteria such as Vibrio (Venkateswaran et al., 1998) and Bacillus spp. (Yamada et al., 1999), was adopted by Kasai et al. (2000) to identify four M. tuberculosis complex members (M. tuberculosis, M. bovis, M. africanum and M. microti). In our study, although proper restriction profiles for all above mentioned four species were observed, the system failed to differentiate $M$. bovis BCG substrains, M. bovis BCG from virulent M. bovis, and M. tuberculosis from $M$. canetti species.

The multiplex PCR described by Bedwell et al. (2001) easily distinguished M. bovis BCG substrains, however failed to differentiate M.tuberculosis, M. africanum, M. canetti, and M.pinnipedii species. Thus, M. bovis BCG Moreau substrain was easily identified in all isolates, determined at the NTLDRI as avirulent ones, however without the value for species identification in virulent isolates.

PCR-based genomic deletion analysis (Warren et al., 2006), described originally as accurate tool for identification of M. canetti, M. tuberculosis, M. africanum, M. microti, M. pinnipedii, M. caprae or M. bovis BCG species, was found the most potent diagnostics option. Regions of differences distinguished M. bovis, M. bovis BCG, M. tuberculosis, M. canetti, M. microti and M. africanum species were described previously (Brosch et al., 2002). Differentiation of M. microti/africanum/pinnipedii species was possible with a second PCR round with additionally designed primers. Although, PCR-RFLP of the partial gyrB gene (Kansai et al., 2000) and multiplex PCR (Bedwell et al., 2001) methods declared improvement of diagnostics potential, only PCR-based genomic deletion analysis developed by Warren et al. (2006), was found capable to precisely and simultaneously differentiate virulent and avirulent mycobacteria or virulent species of M. tuberculosis complex. Multiplex PCR developed by Bedwell et al. (2001) was found a valuable diagnostic option in situations when information on the M. bovis BCG substrain might be needed, eg. in foreigners infected with $M$. bovis BCG.

The criteria for the diagnosis of disseminated BCG infection in people with primary immunodeficiency, including definitive cases confirmed as M. bovis BCG substrain infection by culture and/or standard PCR (Bernatowska et al., 2007) were previously proposed. However, the diagnostic details were not evaluated and discussed. As many different molecular tools were described, their evaluation is expected to improve AEFI surveillance measures. PCR-based genomic deletion analysis might be thus chosen for purposes of rapid identification of mycobacteria carriers, appropriate treatment of patients, epidemiological studies purposes, transmission studies of $M$. bovis between animals and humans, reliable confirmation of $M$. bovis BCG recovered from immunocompromised patients and healthy individuals suspected of AEFI after BCG vaccination.

\section{Acknowledgments}

This study was supported by a grant for scientific research (NN 404207 239) from the Ministry of Science and Higher Education in Poland.

Authors express theirs gratitude to Prof. Dr. Dick van Soolingen from Tuberculosis Reference Laboratory, National Institute for Public Health and the Environment (The Netherlands) who provided M. canetti reference strain.

\section{Literature}

Alexander K.A., P.N. Laver, A.L. Michel, M. Williams, P.D. van Helden, R.M. Warren and N.C. Gey van Pittius. 2010. Novel Mycobacterium tuberculosis complex pathogen, M. mungi. Emerg. Infect. Dis. 16: 1296-1299. 
Augustynowicz-Kopeć E., A. Zabost, S. Brzezińska, M. Wasowicz and Z. Zwolska. 2005. Application of the molecular test PCR multiplex for identification of Mycobacterium bovis BCG strains (in Polish). Pneumonol. Alergol. Pol. 73: 228-233.

Bedwell J., S.K. Kairo, M.A. Behr and J.A. Bygraves. 2001. Identification of substrains of BCG vaccine using multiplex PCR. Vaccine 19: $2146-2151$.

Behr M.A. 2002. BCG-different strains, different vaccines? Lancet Infect Dis. 2: 86-92.

Bernatowska E., B. Wolska-Kusnierz, M. Pac, M. Kurenko-Deptuch, Z. Zwolska, J.L. Casanova, B. Piatosa, J. van Dongen, K. Roszkowski, B. Mikoluc and others. 2007. Disseminated Bacillus Calmette-Guerin infection and immunodeficiency. Emerging. Infect. Dis. 13: 799-801.

Brewer T.F. 2000. Preventing tuberculosis with bacillus CalmetteGuérin vaccine: a meta-analysis of the literature. Clin. Infect. Dis. 31: S64-67.

Brosch R., S.V. Gordon, M. Marmiesse, P. Brodin, C. Buchrieser, K. Eiglmeier, T. Garnier, C. Gutierrez, G. Hewinson, K. Kremer and others. 2002. A new evolutionary scenario for the Mycobacterium tuberculosis complex. Proc. Natl. Acad. Sci. USA 99: 3684-3689. Corbel M.J., U. Fruth, E. Griffiths and I. Knezevic. 2004. Report on a WHO Consultation on the characterisation of BCG strains, Imperial College, London 15-16 December 2003. Vaccine 22: 2675-2680. Cousins D.V., R. Bastida, A. Cataldi, V. Quse, S. Redrobe, S. Dow P. Duignan, A. Murray, C. Dupont, N. Ahmed and others. 2003. Tuberculosis in seals caused by a novel member of the Mycobacterium tuberculosis complex: Mycobacterium pinnipedii sp. nov. Int J. Syst. Evol. Microbiol. 53: 1305-1314.

Gołębiowska M., E. Andrzejewska, I. Stryjewska, H. Baranowska and A. Drążkiewicz. 2008. Advers events following BCG vaccination in infants and children up to 36 months of age (in Polish) Przegl. Epidemiol. 62:71-75.

Jagielski T., E. Augustynowicz-Kopeć and Z. Zwolska. 2010a. Genotyping of Mycobacterium tuberculosis. An overview of the major research techniques (Part I) (in Polish). Pol. Merkur. Lekarski 29: 212-216.

Jagielski T., E. Augustynowicz-Kopeć and Z. Zwolska. 2010b. Genotyping of Mycobacterium tuberculosis. An overview of the major research techniques (Part II) (in Polish). Pol. Merkur. Lekarski 29: 212-216.
Kasai H., T. Ezaki and S. Harayama. 2000. Differentiation of phylogenetically related slowly growing Mycobacteria by their gyr B sequences. J. Clin. Microbiol. 38: 301-308.

Pankowska A. and J. Rożniecki. 1997. Complications after BCG vaccination in children from Urban area of Lodz in 1994-1995 (in Polish). Pneumonol. Alergol. Pol. 65: 761-766.

Rodríguez E., L.P. Sánchez, S. Pérez, L. Herrera, M.S. Jiménez, S. Samper and M.J. Iglesias. 2009. Human tuberculosis due to Mycobacterium bovis and M.caprae in Spain, 2004-2007. Int. J. Tuberc. Lung. Dis. 13: 1536-1541.

Szczuka I. 2002. Adverse events following immunization after BCG vaccination in Poland 1994-2000 (in Polish). Przegl. Epidemiol. 56: 205-216.

Talbot E.A., D.L. Williams and R. Frothingham. 1997. PCR Identification of Mycobacterium bovis BCG. J. Clin. Microbiol. 35: 566-569. Van Ingen J., Z. Rahim, A. Mulder, M.J. Boeree, R. Simeone, R. Brosch, D. van Soolingen. 2012. Characterization of Mycobacterium orygis as M. tuberculosis complex subspecies. Emerg. Infect. Dis. 18: 653-655.

Van Soolingen D., P.W.M. Hermans, P.E.W. de Haas, D.R. Sol and A. van Embden. 1991. The ocurrence and stability of insertion sequences in Mycobacterium tuberculosis complex strains: evaluation of IS dependent DNA polymorphisms as a tool in the epidemiology of tuberculosis. J. Clin. Microbiol. 29: 2578-2586.

Venkateswaran K., N. Dohmoto and S. Harayama. 1998. Cloning and nucleotide sequence of the gyrB gene of Vibrio parahaemolyticus and its application in detection of this pathogen in shrimp. Appl. Environ. Microbiol. 64: 681-687.

Warren R.M., N.C. Gey van Pittius, M. Barnard, A. Hesseling, E. Engelke, M. de Kock, M.C. Gutierrez, G.K. Chege, T.C. Victor, E.G. Hoal and others. 2006. Differentiation of Mycobacterium tuberculosis complex by PCR amplification of genomic regions of difference. Int. J. Tuberc. Lung. Dis. 10: 818-822.

World Health Organization. Global tuberculosis control: WHO report 2011. Available at: http://whqlibdoc.who.int/publications/2011/ 9789241564380_eng.pdf

Yamada S., E. Ohashi, N. Agata, K. Venkateswaran. 1999. Cloning and nucleotide sequence analysis of gyr $\mathrm{B}$ of Bacillus cereus, B. thuringiensis, B. mycoides, and B. anthracis and their application to the detection of B.cereus in rice. Appl. Environ. Microbiol. 65: 1483-1490. 